\title{
Critical Antileishmanial in vitro Effects of Highly Examined Gold Nanoparticles
}

\author{
Muzamil Yaqub Want ${ }^{1,2}$ \\ Priya Yadav ${ }^{1,3}$ \\ Rakin Khan' \\ Garima Chouhan ${ }^{1,4}$ \\ Mohammad Islamuddin 1,5 \\ Sheka Yagub Aloyouni ${ }^{6}$ \\ Asoke P Chattopadhyay ${ }^{7}$ \\ Suliman Yousef AlOmar ${ }^{8}$ \\ Farhat Afrin (ID) ${ }^{1,9}$ \\ 'Parasite Immunology Laboratory, \\ Department of Biotechnology, Jamia \\ Hamdard (Hamdard University), New \\ Delhi, I 0062, India; ${ }^{2}$ Roswell Park \\ Comprehensive Cancer Center, Buffalo, \\ NY, 14263, USA; ${ }^{3}$ Department of \\ Microbiology, University of Kalyani, \\ Kalyani, West Bengal, 74I235, India; \\ ${ }^{4}$ Department of Biotechnology, School of \\ Engineering and Technology, Sharda \\ University, Greater Noida, Uttar Pradesh, \\ 201306, India; ${ }^{5}$ Molecular Virology and \\ Vaccinology Laboratory, Department of \\ Biotechnology, Jamia Millia Islamia, New \\ Delhi, I 10025 , India; ${ }^{6} \mathrm{Health}$ Sciences \\ Research Center, Princess Nourah Bint \\ Abdulrahman University, Riyadh, II67I, \\ Saudi Arabia; ${ }^{7}$ Department of Chemistry, \\ University of Kalyani, Kalyani, West \\ Bengal, 74I235, India; ${ }^{8}$ Doping Research \\ Chair, Department of Zoology, College of \\ Science, King Saud University, Riyadh, \\ I I45I, Saudi Arabia; ${ }^{9}$ Department of \\ Medical Laboratory Technology, Faculty \\ of Applied Medical Sciences, Taibah \\ University, Madina, 4I477, Saudi Arabia
}

Correspondence: Farhat Afrin; Suliman Yousef AIOmar

Tel +9l-7596846639; +966-5007677I7

Email farhatafrin@gmail.com;

syalomar@ksu.edu.sa
Introduction: The current therapeutic armory for visceral leishmaniasis (VL) caused by Leishmania donovani complex is inadequate, coupled with serious limitations. Combination therapy has proved ineffective due to mounting resistance; however, the search for safe and effective drugs is desirable, in the absence of any vaccine. There is a growing interest in the application of nanoparticles for the therapeutic effectiveness of leishmaniasis. Aimed in this direction, we assessed the antileishmanial effect of gold nanoparticles (GNP) against L. donovani in vitro.

Methods: GNP were synthesized and characterized for particle size by dynamic light scattering (DLS) and atomic force microscopy (AFM) and for optical properties by UVvisible spectroscopy. Cytotoxicity of GNP was measured by the MTT proliferation assay. The antileishmanial activity of the nanoparticles was evaluated against $L$. donovani promastigotes and macrophage-infected amastigotes in vitro.

Results: GNP showed a strong SPR peak at $520 \mathrm{~nm}$ and mean particle size, polydispersity index (PDI), and zeta potential of $56.0 \pm 10 \mathrm{~nm}, 0.3 \pm 0.1$ and $-27.0 \pm 3 \mathrm{mV}$, respectively. The GNPs were smooth and spherical with a mean particle diameter of $20 \pm 5 \mathrm{~nm}$. Nanoparticles [1.2-100 $\mu \mathrm{M}$ ] did not reveal any cytotoxicity on RAW 264.7 murine macrophage cell line, but exerted significant activity against both promastigotes and amastigote stages of $L$. donovani with $50 \%$ inhibitory concentrations $\left(\mathrm{IC}_{50}\right)$ of $18.4 \pm 0.4 \mu \mathrm{M}$ and $5.0 \pm$ $0.3 \mu \mathrm{M}$, respectively. GNP showed significant antileishmanial activity with deformed morphology of parasites and the least number of surviving promastigotes after growth reversibility analysis.

Conclusion: GNP may provide a platform to conjugate antileishmanial drugs onto the surface of nanoparticles to enhance their therapeutic effectiveness against VL. Further work is warranted, involving more in-depth mechanistic studies and in vivo investigations.

Keywords: gold nanoparticles, surface plasmon resonance, visceral leishmaniasis L. donovani, promastigotes, amastigotes

\section{Introduction}

Leishmaniasis encompasses a complex spectrum of diseases ranging from cutaneous, mucocutaneous to visceral forms depending on the infecting species. Visceral leishmaniasis (VL) is characterized by prolonged fever, hepatosplenomegaly, substantial weight loss, anemia, pancytopenia, and hypergammaglobulinemia, and is the most severe of the various clinical forms of leishmaniasis. This vectorborne disease is caused by Leishmania donovani complex, which includes L. donovani and Leishmania infantum (also known as Leishmania chagasi in South America). In India, VL or kala-azar is mainly caused by L. donovani species 
and represents $50 \%$ of the global burden of this neglected disease of poverty that can be $100 \%$ fatal in less than two years, if left untreated. In addition, there are reports of outbreaks in low- and non-endemic zones. ${ }^{1-3}$ Treatment of VL relies mainly on pentavalent antimonials, amphotericin B, liposomal amphotericin B, miltefosine, paromomycin or their combinations. ${ }^{4}$ The limitations of present chemotherapeutics include toxicity, noncompliance, prolonged and a cumbersome regimen that is unaffordable to the rural population, increasing antimonial resistance, complications of post kala-azar dermal leishmaniasis (PKDL), and HIV-coinfections. ${ }^{5}$ Combination therapy has proved to be useful over monotherapy in reducing the dose of the drug and hence toxicity; ${ }^{6}$ nonetheless, improved therapy is required in the absence of any vaccine. Furthermore, since the parasites reside mainly in phagolysosomal vacuoles within the macrophages, chemotherapy using present antiparasitics is difficult. Nanoparticulate materials are powerful tools to reach the therapeutic target that is otherwise difficult to attain through common procedures. ${ }^{7}$ Due to the low rate of discovery of antileishmanial drugs, researchers have focused mainly on modulating the delivery of existing drugs. ${ }^{8}$ In the case of leishmaniasis, nanoparticles such as nanoemulsions and liposomes have been shown to be useful as they increase the bioavailability and reduce the toxicity of loaded antileishmanial drugs. ${ }^{9,10}$

Metallic nanoparticles offer promise as an alternative approach to replace or complement the present antibiotics, especially in the therapy of infectious diseases. ${ }^{11,12}$ Studies on the antibacterial action of metal nanoparticles have provided encouraging results. ${ }^{13,14}$ The anti-trypanosomal activity of silver, gold, and platinum nanoparticles has also been explored. ${ }^{15}$ Research on metal nanoparticles and metallic compounds for the treatment of leishmaniasis is gaining ground due to the fact that these compounds inhibit trypanothione metabolism enzymes that are important for the survival of Leishmania. ${ }^{16}$ Auranofin, a drug containing gold has been reported to kill promastigotes of L. infantum by targeting trypanothione reductase. ${ }^{17}$ The inhibitory activity of silver nanoparticles against L. infantum has been reported to be mediated by its effect on trypanothione reductase. ${ }^{18}$ In one investigation, the clinical use of zinc sulfate was found to be as effective as glucantime for the treatment of cutaneous leishmaniasis. ${ }^{19}$ In another study, the antileishmanial activity of platinum compound has been reported against Leishmania major. ${ }^{20}$ The leishmanicidal activity of zinc oxide nanoparticles against Leishmania tropica has also been observed. ${ }^{21}$ Silver and titanium oxide nanoparticles have shown remarkable antileishmanial activity against a few species of Leishmania. ${ }^{22,23}$ The small size and high surface area to volume ratio of nanoparticles allow them to interact with important components of infectious agents especially DNA and enzymes, rendering them nonfunctional. ${ }^{24}$

Gold nanoparticles (GNP) have been widely used as important tools in therapy, drug delivery, targeting, and imaging. ${ }^{25}$ Gold nanoparticles have been reported to exhibit significant activity against $L$. major. ${ }^{26,27}$ Silencing of the gp63 gene in L. major followed by parasite killing has been observed with antisense oligonucleotides hybridized to GNP. ${ }^{28}$ Nanogold with antileishmanial molecules has been reported against $\mathrm{CL}^{29}$ as well as VL. ${ }^{30}$ Different drugs or bioactive compounds have been conjugated with GNP to enhance their delivery to the target site. Surface functionalized GNP via quercetin, ${ }^{31}$ gallic acid ${ }^{32}$ and kaempherol ${ }^{33}$ have shown improved activity against L. donovani. However, in these studies, the activity was tested against axenic amastigotes and macrophageinfected axenic amastigotes. Axenic amastigotes do not represent true intracellular amastigotes within the macrophages. When macrophages are infected with promastigotes that transform into amastigotes, the natural infection is mimicked. Macrophage-infected amastigotes are a more elaborate and physiologic model for drug testing compared to axenic amastigotes. ${ }^{34,35}$ Moreover, activity against axenic amastigotes does not always correlate with efficacy against the parasite in its intracellular niche. $^{34}$

Furthermore, in earlier studies, side effects were observed against peritoneal macrophages harvested from Swiss outbred mice after induction of thioglycollate. Inflammatory thioglycollate potentiates the activation of macrophages. Therefore, RAW 264.7 murine macrophage cells were used as model phagocytes in our study. There are also reports on the activity of green synthesized GNP against $L$. donovani ${ }^{36}$ but, the activity was evaluated against the promastigote forms alone. An effective antileishmanial drug should be active against both the morphological stages of the parasite. Furthermore, loss of activity of biosynthesized nanoparticles has also been reported. ${ }^{37,38}$ At present, there are no reports on the antileishmanial effect of GNP alone against $L$. donovani promastigotes and amastigotes in vitro. In this study, we evaluated the antileishmanial potential of GNP against 
L. donovani promastigotes and macrophage-infested amastigotes.

\section{Materials and Methods}

\section{Animals and Cell Lines}

Six-eight weeks old female BALB/c mice (weighing about 25-30 g), obtained from the Central Animal House Facility, Jamia Hamdard, were used to maintain the Leishmania parasites. Ethics approval for the study (Approval No. 458) was sought from Jamia Hamdard Animal Ethics Committee, which followed the guidelines laid down by the Committee for the Purpose of Control and Supervision of Animal Experiments, Ministry of Empowerment and Social Justice, Government of India. The use of the murine macrophage cell line, RAW 264.7 (a kind gift from Dr. B.S. Dwarakanath, INMAS, New Delhi), had approval from the ethics review board.

\section{Parasite Culture}

The Indian strain of L. donovani (MHOM/IN/83/AG83) was routinely passaged in BALB/c mice and, after transformation, was cultured at $22^{\circ} \mathrm{C}$ in M199 supplemented with $10 \%$ heat-inactivated FBS, HEPES (25 mM), L-glutamine (2 $\mathrm{mM})$, penicillin $\mathrm{G}\left(100 \mathrm{IU} \mathrm{mL}^{-1}\right)$ and streptomycin $(100$ $\left.\mu \mathrm{g} \mathrm{m}^{-1}\right)$ at an average density of $2 \times 10^{6}$ cells $\mathrm{mL}^{-1} .{ }^{39}$

\section{Synthesis of Gold Nanoparticles (GNPs)}

GNPs were synthesized by the microwave-assisted heating method via a chemical reduction wherein, trisodium citrate dihydrate was used to reduce the $\mathrm{HAuCl}_{4} \cdot 3 \mathrm{H}_{2} \mathrm{O}$ solution. ${ }^{40}$ Briefly, $1 \mathrm{mM}$ tetrachloroaurate $\left(\mathrm{HAuCl}_{4} \cdot 3 \mathrm{H}_{2} \mathrm{O}\right)$ solution was brought to a rolling boil in a microwave to which $1 \%$ trisodium citrate dihydrate was quickly added in a 10:1 ratio. The change in the colour of the suspension from pale yellow to brilliant red indicated the successful synthesis of GNP. The solution was cooled and then subjected to ultracentrifugation to wash off the free citrate. The nanoparticle pellet was resuspended in nano-pure water and characterized by UV-visible spectroscopy, dynamic light scattering (DLS), and atomic force microscopy (AFM).

\section{Characterization of Gold Nanoparticles (GNPs)}

Surface Plasmon Resonance (SPR) Determination by UltraViolet (UV)-Visible Spectroscopy

The synthesized GNPs were analysed spectrophotometrically at wavelength ranging from 200 to $800 \mathrm{~nm}$ using a UV-Visible spectrophotometer (Lamda 20, PerkinElmer) to determine the characteristic properties of surface plasmon resonance (SPR). ${ }^{41}$ The GNP concentration was adjusted to give an absorbance of less than 1 . The synthesized GNPs were further used for antileishmanial studies.

\section{Particle Size Distribution and Zeta Potential by Dynamic Light Scattering (DLS)}

The mean particle size and polydispersity index (PDI) of GNPs were estimated in a Malvern Zeta Sizer (Nano ZS, Malvern Instruments Inc. Worcestershire, UK) using the DLS technique at a scattering angle of $90^{\circ}$ at $25^{\circ} \mathrm{C}$. The sample of GNP was diluted with Milli-Q water and analyzed in triplicate.

The zeta potential $(\zeta)$ of the nanoparticles was also measured by a zetasizer at room temperature. The diluted GNPs were placed in an electrophoretic cell and the data were acquired in triplicate.

\section{Surface Morphology of Gold Nanoparticles (GNP) Using Atomic Force Microscopy}

The surface morphology, shape and size of GNP were further visualized by the atomic force microscope (AFM, Veeco, Innova) in contact mode with the help of the silicon nitride nano probe cantilever, at a spring constant of 49 $\mathrm{N} \mathrm{m}^{-1}{ }^{41}$ The obtained images were further analyzed and processed by Veeco SPM Lab analysis software.

\section{In vitro Analysis of Cytotoxicity of Gold Nanoparticles (GNPs)}

The cytotoxicity of GNP was evaluated in the RAW 264.7 murine macrophage Cell line using MTT assay. ${ }^{42}$ In brief, $2 \times 10^{5}$ Cells per well in RPMI 1640 medium were seeded in a flat bottom 96-well tissue culture plate for $24 \mathrm{~h}$ at $37^{\circ}$ $\mathrm{C}$ in a $\mathrm{CO}_{2}$ incubator. The cells were incubated in triplicate with serial three-fold dilutions of GNP or pentamidine, starting from $100 \mu \mathrm{M}$ at $37^{\circ} \mathrm{C}, 5 \% \mathrm{CO}_{2}$. Macrophages without GNP or pentamidine were left as negative control. After $48 \mathrm{~h}$ of incubation, MTT $\left(5 \mathrm{mg} \mathrm{mL}^{-1}\right)$ was added to each well and on the formation of formazan crystals, the plate was centrifuged $\left(2100 \times \mathrm{g}, 20 \mathrm{~min}, 4^{\circ} \mathrm{C}\right)$, the supernatant carefully aspirated, and the crystals so formed were dissolved using DMSO:isopropanol (1:1). Using a microplate reader (Spectra max 450), the plate was read at $570 \mathrm{~nm}$, with $690 \mathrm{~nm}$ as background. The result was represented as a percentage of viable Cells compared to the negative control and was calculated as follows: 


$$
\begin{aligned}
& \text { Cell viability }(\%) \\
& \quad \begin{array}{l}
\text { Mean absorbance of treated sample }(570 \mathrm{~nm}) \\
=\frac{- \text { Mean absorbance of treated sample }(690 \mathrm{~nm})}{\text { Mean absorbance of untreated sample }(570 \mathrm{~nm})} \times 100 \\
\quad \text { - Mean absorbance of untreated sample }(690 \mathrm{~nm})
\end{array}
\end{aligned}
$$

\section{In vitro Antileishmanial Activity of Gold} Nanoparticles (GNPs) Against L. donovani

\section{Promastigotes}

The growth kinetics of $L$. donovani promastigotes was studied by incubating promastigotes $\left(2 \times 10^{6}\right.$ cells $\left.\mathrm{mL}^{-1}\right)$ with GNP $(75 \mu \mathrm{M})$ in M199 medium supplemented with $10 \%$ FBS (complete medium). Pentamidine was added as a reference drug, while parasites in medium alone, without any drug or GNP, served as a control. Viable parasites were microscopically counted for 5 days and parasites without any motility were marked dead. ${ }^{43}$

To further confirm the leishmanicidal effect of GNPs, promastigotes after 7 days in culture, with or without treatment, were analyzed using a growth reversibility assay. Briefly, promastigotes were subjected to drug withdrawal by washing twice with incomplete M199 medium (without FBS) and then resuspended in fresh complete M199 medium at $22^{\circ} \mathrm{C}$ for a further 4 days. The viable parasites were counted using a phase-contrast microscope with a $40 x$ objective. ${ }^{43}$

\section{Evaluation of the $50 \%$ Inhibitory Concentration $\left(\mathrm{IC}_{50}\right)$ of Gold Nanoparticles (GNPs) for L. donovani Promastigotes}

L. donovani promastigotes were incubated with serial twofold dilutions of GNP $(0-25 \mu \mathrm{M})$ and cultured at $22^{\circ}$ C. Pentamidine was added as a standard antileishmanial drug. The untreated $L$. donovani promastigotes served as a parasite control. After 4 days, the parasites were enumerated microscopically using a phase contrast microscope and the percent viability determined ${ }^{43}$ according to the formula:

$$
\begin{aligned}
& \text { Viability }(\%) \\
& =\frac{\text { Average number of treated promastigotes per } \mathrm{mL}}{\text { Average number of untreated promastigote per } \mathrm{mL}} \times 100
\end{aligned}
$$

The $\mathrm{IC}_{50}$ of GNP on promastigotes was calculated using a linear regression analysis.

\section{Evaluation of Promastigote Morphology After Gold Nanoparticles (GNPs) Treatment}

Morphology of $L$. donovani promastigotes with and without GNP treatment was studied after 4 days in culture by enumeration under a phase-contrast microscope with $40 \mathrm{x}$ objective. For each sample, a minimum of 10 microscopic fields were observed and the images were taken using NIS-Elements imaging software. ${ }^{44}$

\section{Anti-Amastigote Effect of Gold Nanoparticles (GNPs) ex vivo}

L. donovani promastigotes were cultured in RPMI 1640 medium prior to infecting the RAW 264.7 macrophage cell line. Briefly, $5 \times 10^{5}$ macrophages per cover slip were seeded in a 24-well tissue culture plate and left to adhere overnight in a $\mathrm{CO}_{2}$ incubator at $37^{\circ} \mathrm{C}$. Adherent macrophages were incubated with stationary phase $L$. donovani promastigotes in a cell to parasite ratio of $1: 10$, for at least $12 \mathrm{~h}$ at $37^{\circ} \mathrm{C}, 5 \% \mathrm{CO}_{2}$. Non-phagocytosed promastigotes were gently aspirated by washing with incomplete RPMI 1640 medium (without FBS) and infected macrophages were further incubated with GNP $(1-15 \mu \mathrm{M})$ for $48 \mathrm{~h}$ at $37^{\circ} \mathrm{C}, 5 \% \mathrm{CO}_{2}$. Infected macrophages that were not treated were left as a control. The cover slips were then fixed, Giemsa stained and a minimum of 200 macrophages per cover slip were microscopically enumerated to determine the number of infected macrophages and the number of intracellular amastigotes. ${ }^{42}$ The mean percent infection was calculated using the following formula:

Infection $(\%)$
$=\frac{\text { Number of amastigotes per } 200 \text { macrophages (treated group) }}{\text { Number of amastigotes per } 200 \text { macrophages (infected group) }} \times 100$

The $\mathrm{IC}_{50}$ of GNP on amastigotes was determined using a linear regression analysis. The percentage of infected macrophages was also analyzed according to the following formula:

Infected macrophage $(\%)$

Number of infected macrophage per 200 macrophages

$=\frac{\text { (treated group) }}{\text { Number of infected macrophages per } 200 \text { macrophages }} \times 100$

(infected control group)

\section{Statistical Analysis}

All data were represented as mean \pm standard error (SE) of samples in triplicate and were from one of three 
independent experiments. Statistical analysis was performed by one-way analysis of variance (ANOVA) using the graph pad InStat. $P$-values of $<0.05$ were considered statistically significant.

\section{Results}

\section{Synthesis and Characterization of Gold Nanoparticles (GNPs)}

GNPs were successfully synthesized via a simple chemical reduction by mixing an aqueous solution of trisodium citrate dihydrate with a microwave-heated $\mathrm{HAuCl}_{4}$ solution. A brilliant red suspension of GNPs was formed after complete reduction of $\mathrm{Au}^{3+}$ ions to $\mathrm{Au}^{0}$ during the reaction. UV-visible absorption spectra revealed the presence of a prominent SPR peak at $520 \mathrm{~nm}$, indicating the formation of spherical GNPs (Figure 1A). The position of SPR peak varies depending on the particle size, shape, and dielectric constant of the surrounding medium. The mean
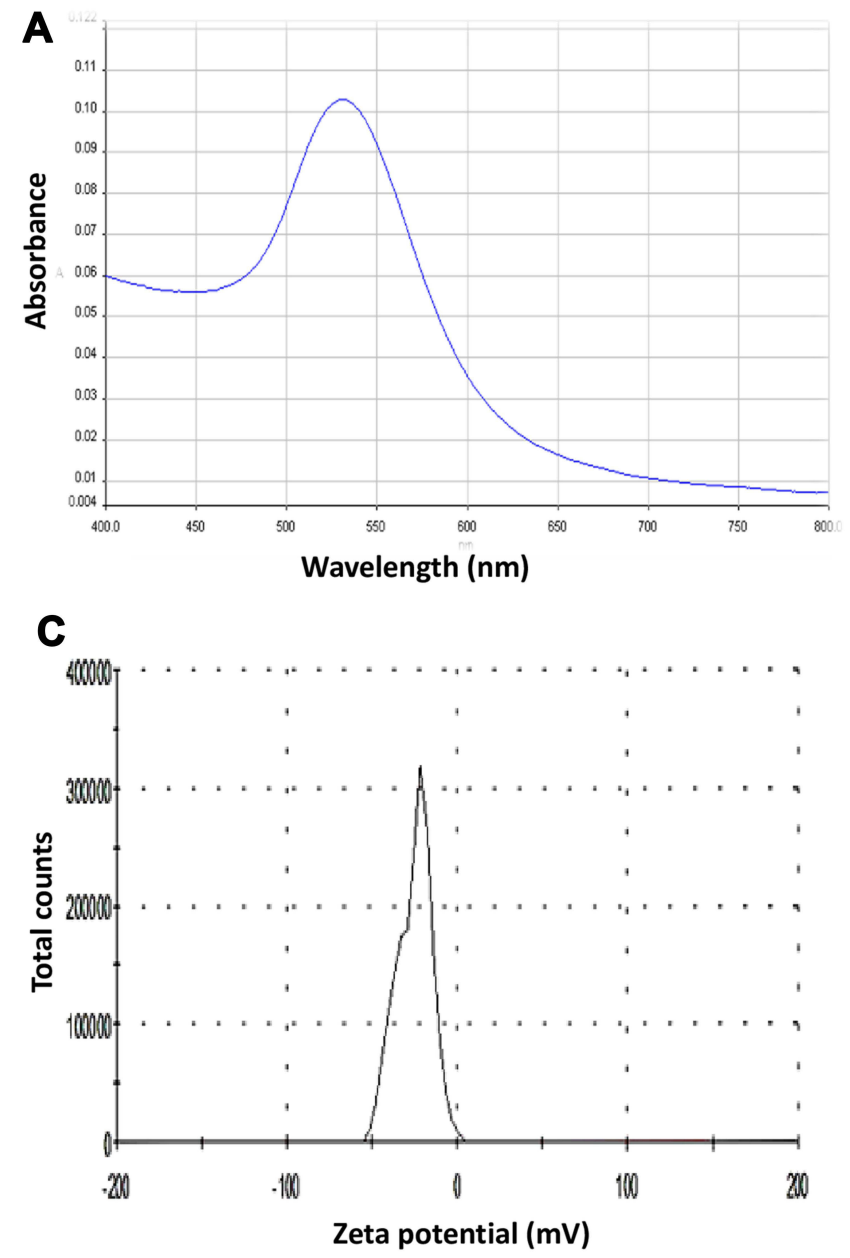

hydrodynamic diameter and PDI of GNP were $56.0 \pm 10$ $\mathrm{nm}$ and $0.3 \pm 0.1$, respectively, as determined by DLS (Figure 1B). The zeta potential, which dictates the stability of GNP, was found to be $-27 \pm 3 \mathrm{mV}$ (Figure 1C). The presence of uniform negative charge on the surface of synthesized GNP imparts high stability to these nanoparticles, thus preventing their aggregation. For further morphological analysis, GNPs were vacuum dried onto clean glass round cover slips $(18 \mathrm{~mm})$ and observed under AFM. The AFM images revealed that the synthesized GNP had an average particle size of $20 \pm 5 \mathrm{~nm}$ and were spherical with a smooth surface (Figure 1D).

\section{In vitro Cytotoxicity of Gold Nanoparticles (GNPs)}

The dose-dependent cytotoxicity of the synthesized GNP in macrophage cell lines (RAW 264.7) was assessed using the MTT assay. The cells were incubated with different

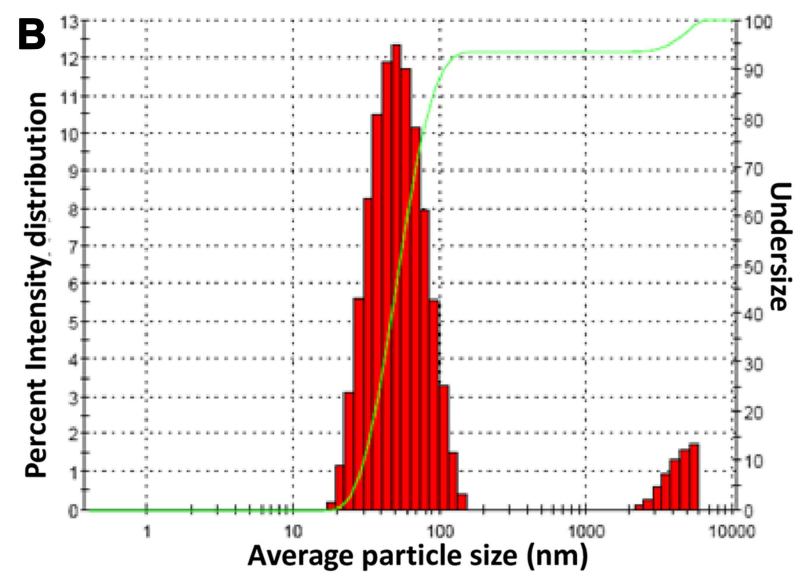

D

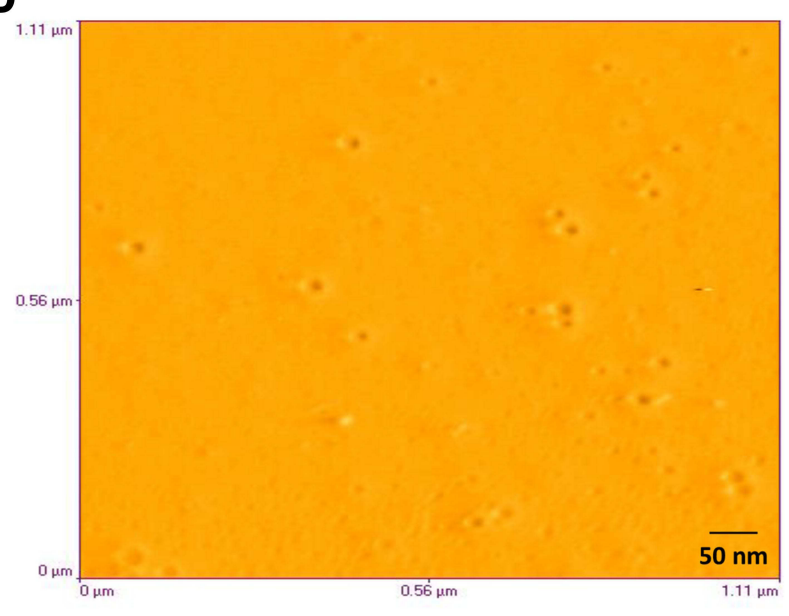

Figure I Characterization of GNP (A) absorption spectra showing the surface plasmon resonance peak, (B) size distribution pattern, (C) zeta potential measurement graph, (D) microscopic image showing the morphological depictions of nanoparticles by AFM at contact mode. 
concentrations of GNP for $48 \mathrm{~h}$ at $37^{\circ} \mathrm{C}, 5 \% \mathrm{CO}_{2}$ while the pentamidine (as a reference antileishmanial drug) treated cells served as a positive control. The untreated RAW 264.7 cells (negative control) showed 100\% formazan crystal formation upon subsequent addition of MTT. GNP exhibited negligible toxicity even at $100 \mu \mathrm{M}$ unlike pentamidine, resulting in a significant decrease in cell viability (Figure 2). The cytotoxicity data revealed that GNPs have excellent biocompatibility in macrophage cell lines.

\section{Antileishmanial Effect of Gold Nanoparticles (GNP) on $L$. donovani \\ Promastigotes}

Time-Dependent Killing of $L$. donovani Promastigotes The growth kinetics of $L$. donovani promastigotes were studied after incubation with GNPs and pentamidine for 5 days. L. donovani growth decreased significantly and cell density reached zero within 5 days of incubation with GNPs $(75 \mu \mathrm{M})$, whereas all parasites were not-motile within $48 \mathrm{~h}$ after pentamidine treatment. The untreated parasites proliferated at a normal rate (Figure $3 \mathrm{~A}$ ).

\section{Growth Reversibility Assay}

Seven days after incubation with GNPs, the parasites were washed and cultured in fresh M199 medium for a further 4 days. The GNP-treated parasites showed a slight reversal of growth, whereas the untreated control promastigotes exhibited visible reversibility. This confirmed the

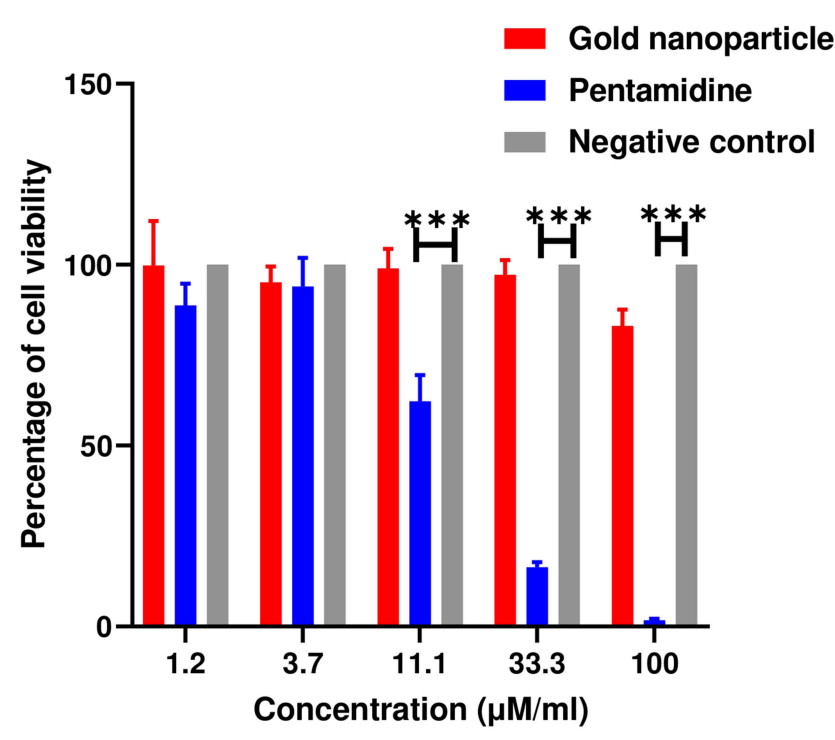

Figure 2 Cytotoxicity of gold nanoparticle on RAW 264.7 macrophage cell line by MTT assay. Pentamidine was used as a reference antileishmanial drug. Macrophages alone served as a negative control. Data $(n=3)$ are represented as mean \pm SE. Significance is calculated as $* * * P<0.001$, pentamidine versus negative control. antileishmanial effect of GNP as also observed with pentamidine (Figure 3B).

Dose-Dependent Killing of $L$. donovani Promastigotes GNPs demonstrated a dose-dependent activity on L. donovani promastigotes. The $50 \%$ inhibitory concentration $\left(\mathrm{IC}_{50}\right)$ was calculated by linear regression analysis of the plot between percent viability versus nanoparticle concentration after 4 days of incubation of promastigotes with GNPs or pentamidine (Figure $3 \mathrm{C}$ ). The $\mathrm{IC}_{50}$ of GNP and pentamidine in promastigotes was found to be $18.4 \pm 0.4$ $\mu \mathrm{M}$ and $3.5 \pm 0.2 \mu \mathrm{M}$, respectively (Table 1 ).

\section{Morphology of Gold Nanoparticles (GNP)-Treated Promastigotes}

The cellular morphology of the parasites studied by phase contrast microscopy confirmed that the promastigotes were shrunken, round, and non-motile upon incubation with GNPs and pentamidine in contrast to the untreated control parasites that were elongated, slender, and motile (Figure 3D).

\section{Antileishmanial Activity of Gold Nanoparticles (GNP) Against L. donovani Amastigotes}

The effect of GNP on macrophage-infected amastigotes was investigated and compared with the standard antileishmanial drug pentamidine. The percent infection by amastigote (with respect to the infected control) upon treatment with GNPs is presented in Figure 4A. GNPs were effective against the amastigote form of the parasites with $\mathrm{IC}_{50}$ of $5.0 \pm 0.3 \mu \mathrm{M}$, which was close to that of pentamidine having $\mathrm{IC}_{50}$ of $1.5 \pm$ $0.7 \mu \mathrm{M}$ (Table 1). GNP at a concentration of $15 \mu \mathrm{M}$ reduced infected macrophages to around $10 \%$ compared to untreated infected control (Figure 4B).

\section{Discussion}

There is a dire need to develop new therapeutic modalities for the treatment of VL. Exploring nanoparticles is one way to overcome the problem of drug resistance and toxicity of antileishmanial compounds on healthy cells. ${ }^{10,45}$ The high surface area to volume ratio of nanoparticles may lead to an increase in the area of contact with parasites to mediate the antileishmanial effect. ${ }^{24}$ The limitations of current therapeutic drugs coupled with the absence of any effective vaccine and the remarkable activity of GNP against various Leishmania species $^{27,28,46}$ prompted us to investigate its potential against L. donovani. Few studies have investigated the efficacy 

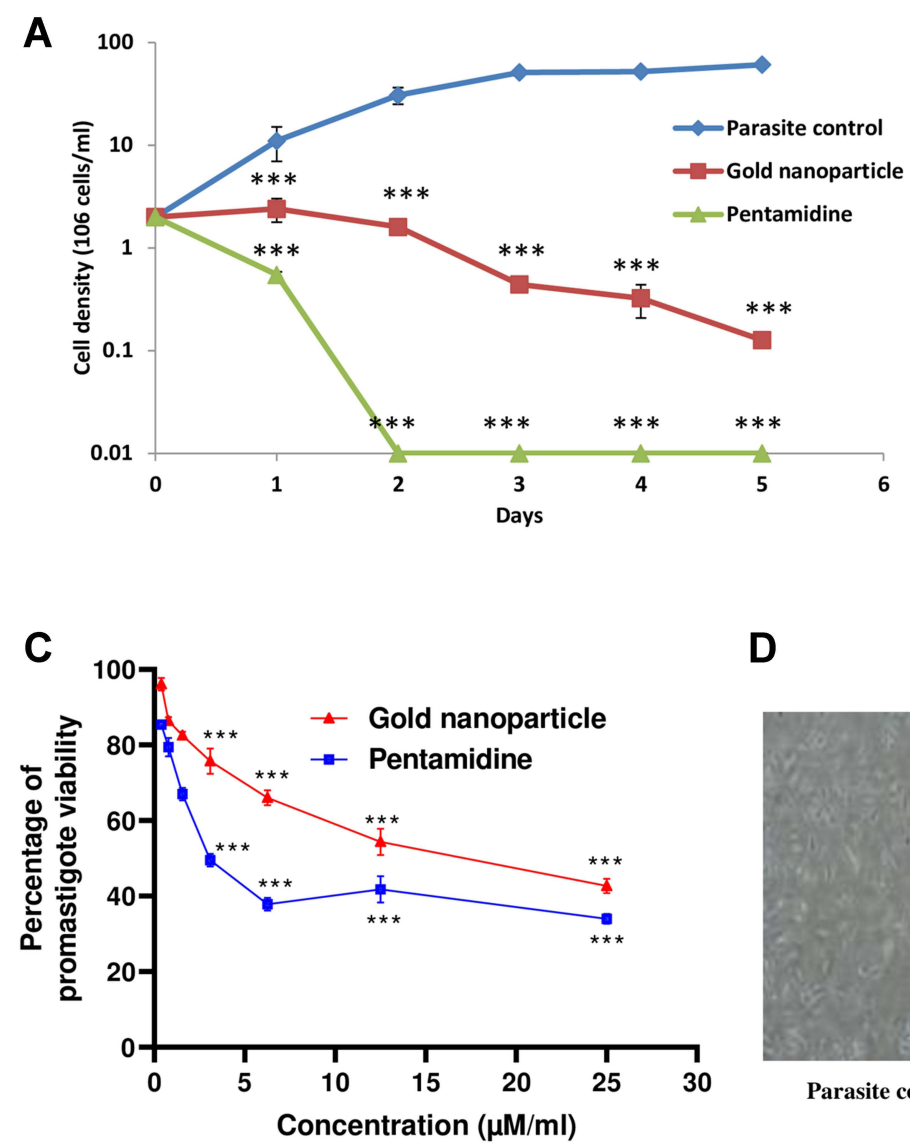

B

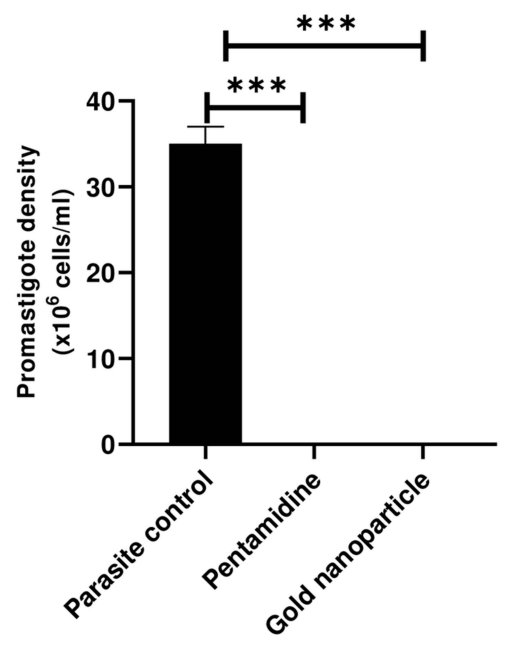

D

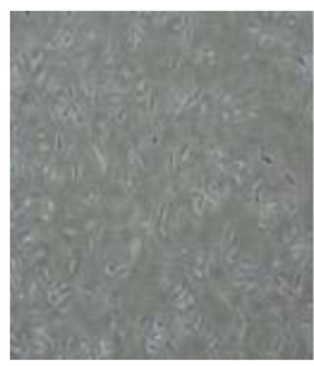

Parasite control

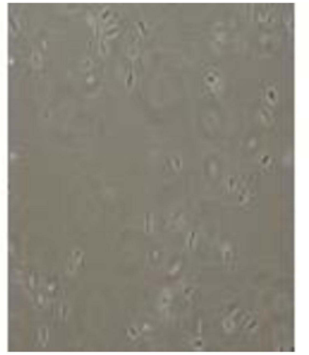

Gold nanoparticle

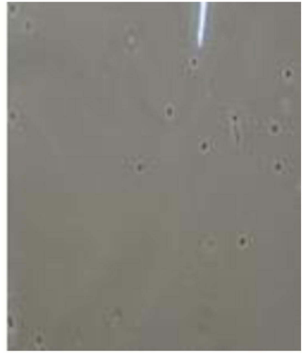

Pentamidine

Figure 3 Antileishmanial activity of gold nanoparticles against L. donovani. (A) Time-dependent activity of gold nanoparticle or pentamidine (75 $\mu M)$ against promastigotes compared to untreated parasite control, (B) growth reversibility assay at 4 days in fresh medium after the withdrawal of spent culture medium, (C) dose-dependent killing of L. donovani promastigotes by gold nanoparticles or pentamidine, (D) cellular morphology of treated promastigotes at $40 \times$ magnification. All data ( $n=3$ ) are represented as mean \pm SE. Significance is represented as $* * * P<0.001$ versus untreated parasite control.

of GNP against the visceralizing species, L. donovani. In one study, it has been tested against the axenic amastigotes $^{32}$ and in another against the promastigotes. ${ }^{36}$ However, the intracellular macrophage-amastigote model is so far the gold standard in the search for in vitro antileishmanial drug screening, as it involves the host cellmediated effects, ${ }^{47}$ including macrophage's microbicidal activities. ${ }^{48}$ With axenic amastigotes, the natural niche of the parasite is absent, unlike the macrophage-infected

Table I Effect of GNP on $L$. donovani Promastigotes and Amastigotes

\begin{tabular}{|l|c|c|}
\hline $\begin{array}{l}\text { Test } \\
\text { Sample }\end{array}$ & $\begin{array}{c}\text { IC }_{\mathbf{5 0}}(\boldsymbol{\mu M}) \\
\text { Promastigotes }\end{array}$ & $\begin{array}{c}\text { IC } \\
\text { Amastigotes }\end{array}$ \\
\hline GNP & $18.4 \pm 0.4$ & $5.0 \pm 0.3$ \\
Pentamidine & $3.5 \pm 0.2$ & $1.5 \pm 0.7$ \\
\hline
\end{tabular}

Notes: IC $C_{50}$ values of GNP and pentamidine (a reference antileishmanial drug) on the promastigote and amastigote forms of $L$. donovani are shown. Data $(n=3)$ is represented as mean $\pm \mathrm{SE}$. amastigotes. ${ }^{49}$ Pentavalent antimonials have been reported to show antileishmanial activity only against intracellular amastigotes, as reduction to trivalent antimonials, critical for leishmanicidal activity, occurs only with fully differentiated intramacrophagic amastigotes. ${ }^{50}$ On the contrary, free-living axenic amastigotes have been reported to retain some promastigote-like traits and therefore require continuous monitoring for amastigote-like features and have also been found to contribute to high false-positive rates in drug sensitivities. ${ }^{50}$ Furthermore, the $\mathrm{IC}_{50}$ values for intramacrophagic amastigotes have been reported to be much higher than those for the axenic amastigotes. ${ }^{34,35}$

In this study, we synthesized and characterized citratestabilized GNP and assessed its potential as an antileishmanial agent in vitro against $L$. donovani. The GNP showed a strong surface plasmon band at $520 \mathrm{~nm}$ that falls in the visible range, thus confirming the synthesis of nanoparticles. $^{51}$ The hydrodynamic diameter, PDI and zeta potential of the synthesized GNP were $56.0 \pm 10 \mathrm{~nm}, 0.3 \pm$ 

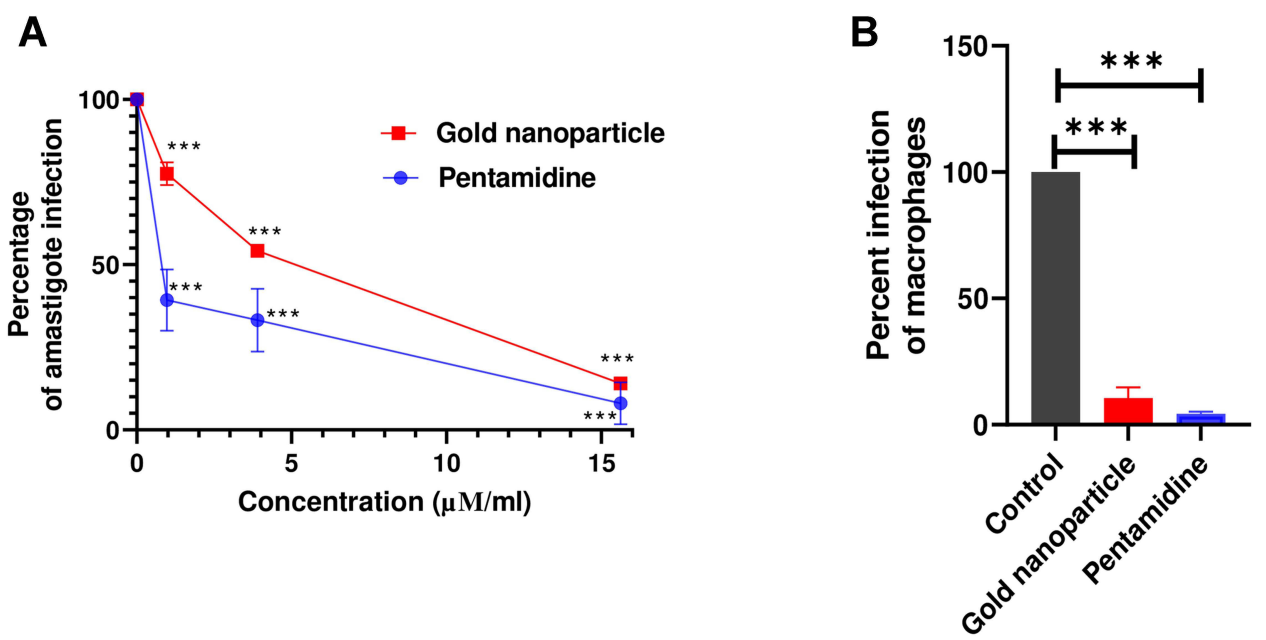

Figure 4 Antileishmanial activity of the gold nanoparticles against $L$. donovani amastigotes $(\mathbf{A})$ dose-dependent activity of gold nanoparticle or pentamidine on $L$. donovani amastigotes presented as amastigote infectivity (percentage of infected control), $(\mathbf{B})$ infected macrophages (percentage of infected control) at I5 $\mu M$ GNP. All the data $(n=3)$ are represented as mean $\pm \mathrm{SE}$. $* * * p<0.001$ with respect to the untreated infected control.

0.1 and $-27.0 \pm 3 \mathrm{mV}$, respectively. The zeta sizer measures the hydrodynamic size; therefore, to reveal the accurate size of GNPs, atomic force microscopy was carried out. The AFM data revealed that the GNPs were spherical in shape, having a smooth morphology and the average size of the GNPs was around $20 \pm 5 \mathrm{~nm}$, that is, about two times smaller when analyzed by AFM compared to DLS.

The important finding is that GNP exerted significant antileishmanial activity against extracellular promastigotes and intracellular amastigotes. There was a decrease in the proliferation of L. donovani promastigotes upon incubation with increasing concentrations of nanoparticles. GNPs inhibited promastigote growth in a time-dependent manner and the number of parasites approached zero within 5 days, in contrast to untreated control promastigotes that proliferated at a normal rate. The treated promastigotes after the withdrawal of GNPs and culture in fresh M199 medium for 4 days showed scrimpy growth compared to the untreated promastigotes, corroborating the antileishmanial effect of GNP, as also observed with pentamidine. Pentamidine, the second line of treatment for the various forms of leishmaniasis, was used as the reference drug in our studies. Phase-contrast microscopic images of L. donovani promastigotes treated with GNP demonstrated certain morphological changes that were very similar to the signs of programmed cell death. ${ }^{23}$ The treated promastigotes became aflagellated, oval, or round and shrunken in contrast to the untreated parasites. We also demonstrated a potent activity of GNPs against intracellular amastigotes of L. donovani $\left(\mathrm{IC}_{50} 5.0 \pm 0.3 \mu \mathrm{M}\right)$, which was 3.6 times less compared to that on promastigotes $\left(\mathrm{IC}_{50} 18 \pm 0.4\right.$ $\mu \mathrm{M})$. Thus, the amastigote forms of the parasites were more susceptible to GNPs.

It is a well-established fact that the uptake of GNPs, as well as toxicity within the cells, depends on the size, shape, and surface functionality. In the case of macrophages, endocytosis is the most effortless and energetically favored pathway for uptake of nanoparticles. Receptormediated endocytosis has been reported for the entry and transport of GNPs into cells. ${ }^{52}$ The "rule of thumb" states that particles larger than $200 \mathrm{~nm}$ are internalized through phagocytosis, while particles smaller than $200 \mathrm{~nm}$ enter through micropinocytosis. ${ }^{53}$ Since the synthesized GNPs in our study are less than $100 \mathrm{~nm}$, they are likely to enter macrophages via pinocytosis. ${ }^{54}$ However, phagocytosis or pinocytosis, or both, may have been involved in the uptake of GNPs. ${ }^{55}$

Additionally, it was observed that macrophages exposed to GNPs did not generate nitric oxide (NO), the major microbicidal molecule (data not shown). Gold atoms are known to exhibit a low reactivity, in particular, regarding oxidation by dissolved oxygen. Thus, less free ions are released from GNPs and fewer reactive oxygen species (ROS) are generated. Consequently, ROS generation and ion release are supposed to play a subordinate role in activity of GNPs, while direct interaction with the cell membrane and binding to intracellular components may represent the key mechanisms of GNP activity. ${ }^{56}$ The absence of ROS has also been observed in the GNP-mediated killing of bacterial cells. ${ }^{57} \mathrm{On}$ the contrary, the NO-mediated immunomodulatory effect of 
L. amazonensis-infected macrophages has been reported to induce leishmanicidal activity by silver nanoparticles. ${ }^{23}$ Bimetallic gold-silver nanoparticles have been found to induce antileishmanial activity against $L$. donovani by apoptosis-like death and ROS generation. ${ }^{58}$ We conclude ROSindependent killing of Leishmania parasites by GNPs, which may be mediated by apoptosis.

Direct adherence of nanoparticles to the cell surface causing disruption of cell membrane has also been suggested. ${ }^{56}$ GNPs have been reported to damage proteins and organelles after cellular entry. GNPs may also induce the secretion of hypoxic macrophages. In the absence of oxygen, GNPs can aggregate within macrophages, lowering cell proliferation and can induce the activation of macrophage inflammasome, which promotes the inflammatory response. ${ }^{59}$ The high surface area-to-volume ratio and the small size of the nanoparticles enhances their interaction with microbes and thus mediate a wide range of antimicrobial effects. ${ }^{60}$ The probable mechanism of antileishmanial activity of GNP may be due to the surface area of GNP in contact with $L$. donovani promastigotes and amastigotes, which can damage the membrane or bind to proteins or DNA, making them non-functional. ${ }^{61}$ The enhanced activity of GNP towards amastigotes may be due to more interaction of GNP with the cell surface receptors of amastigotes than with those of promastigotes. Our results also demonstrated that GNPs are devoid of cytotoxicity in the RAW 264.7 macrophage cell line. The ROS-independent mechanism of action of GNP partly explains the non-toxicity of GNP to mammalian cells. ${ }^{62,63}$

Thus, our study confirms the dose and time-dependent antileishmanial effect of GNP against both forms of L. donovani: the promastigotes in vitro and intracellular amastigotes ex vivo. GNP stands out as a new therapeutic option that can be considered for future in vivo investigations, depicting a potential treatment for VL. The leishmanicidal potential of functionalized silver nanoparticles also opens the door for the functionalization of GNPs. ${ }^{64}$ The construction of nanogold with antileishmanial molecules could represent another effective strategy, as has been reported for CL. ${ }^{29}$ Further,; the synergistic antileishmanial effect of nanoassemblies of chitosan-titanium dioxide loaded with glucantime, ${ }^{46}$ and Amphotericin B conjugated GNPs $^{65}$ also open the possibility of exploring GNPs to complement existing antileishmanial chemotherapy at lower doses of drugs.

\section{Conclusions}

The GNP synthesized by the microwave-assisted heating method was evaluated for its activity against $L$. donovani and toxicity on the macrophage cell line. The ROS-independent antileishmanial activity of GNP may have been mediated via parasite membrane disruption and/or apoptosis, which needs to be explored. Our in vitro results encourage the study of GNP against $L$. donovani in vivo and may have prospects in therapeutic applications of functionalized and/or drug conjugated GNP. However, a deeper understanding of the biological effects of GNPs is required to develop safe and effective nanomedicines.

\section{Acknowledgment}

The authors thank Prof. T. Basu at the Department of Biochemistry, University of Kalyani, West Bengal, for providing the facility of the Zeta sizer and AFM. The authors are grateful to the Deanship of Scientific Research, King Saud University for funding through Vice Deanship of Scientific Research Chairs. This research was also funded by the Deanship of Scientific Research at Princess Nourah bint Abdulrahman University through the Fast-track Research Funding Program.

\section{Disclosure}

The authors report no conflicts of interest in this work.

\section{References}

1. Kumar A, Saurabh S, Jamil S, Kumar V. Intensely clustered outbreak of visceral leishmaniasis (kala-azar) in a setting of seasonal migration in a village of Bihar, India. BMC Infect Dis. 2020;20(1):10. doi:10.1186/s12879-019-4719-3

2. Mandal R, Kesari S, Kumar V, Das P. Trends in spatio-temporal dynamics of visceral leishmaniasis cases in a highly-endemic focus of Bihar, India: an investigation based on GIS tools. Parasit Vectors. 2018;11(1):220. doi:10.1186/s13071-018-2707-x

3. Kumari S, Dhawan P, Panda PK, Bairwa M, Pai VS. Rising visceral leishmaniasis in Holy Himalayas (Uttarakhand, India) - a cross-sectional hospital-based study. J Family Med Prim Care. 2020;9(3):1362. doi:10.4103/jfmpc.jfmpc_1174_19

4. van Griensven J, Diro E. Visceral leishmaniasis: recent advances in diagnostics and treatment regimens. Infect Dis Clin. 2019;33(1):79-99.

5. Srivastava S, Mishra J, Gupta AK, Singh A, Shankar P, Singh S. Laboratory confirmed miltefosine resistant cases of visceral leishmaniasis from India. Parasit Vectors. 2017;10(1):49. doi:10.1186/s13071017-1969-z

6. Goswami RP, Rahman M, Das S, Tripathi SK, Goswami RP. Combination therapy against Indian visceral Leishmaniasis with Liposomal Amphotericin B (FungisomeTM) and short-course miltefosine in comparison to miltefosine monotherapy. Am J Trop Med Hyg. 2020;103:308.

7. Bruni N, Stella B, Giraudo L, Della Pepa C, Gastaldi D, Dosio F. Nanostructured delivery systems with improved leishmanicidal activity: a critical review. Int J Nanomed. 2017;12:5289. doi:10.2147/IJN.S140363 
8. de Souza A, Marins DSS, Mathias SL, et al. Promising nanotherapy in treating leishmaniasis. Int $J$ Pharm. 2018;547(1-2):421-431. doi:10.1016/j.ijpharm.2018.06.018

9. Dar MJ, Din FU, Khan GM. Sodium stibogluconate loaded nano-deformable liposomes for topical treatment of leishmaniasis: macrophage as a target cell. Drug Deliv. 2018;25(1):1595-1606. doi:10.1080/10717544.2018.1494222

10. Ray L, Karthik R, Srivastava V, et al. Efficient antileishmanial activity of amphotericin B and piperine entrapped in enteric coated guar gum nanoparticles. Drug Deliv Transl Res. 2021;11:118-130.

11. Allahverdiyev AM, Abamor ES, Bagirova M, et al. Antileishmanial effect of silver nanoparticles and their enhanced antiparasitic activity under ultraviolet light. Int $J$ Nanomedicine. 2011;6:2705-2714. doi: $10.2147 /$ IJN.S23883

12. Liao S, Zhang Y, Pan X, et al. Antibacterial activity and mechanism of silver nanoparticles against multidrug-resistant Pseudomonas aeruginosa. Int J Nanomed. 2019;14:1469. doi:10.2147/IJN.S191340

13. Zhou Y, Kong Y, Kundu S, Cirillo JD, Liang H. Antibacterial activities of gold and silver nanoparticles against Escherichia coli and bacillus Calmette-Guerin. J Nanobiotechnol. 2012;10:19. doi:10.1186/1477-3155-10-19

14. Araujo EA, Andrade NJ, da Silva LH, et al. Antimicrobial effects of silver nanoparticles against bacterial cells adhered to stainless steel surfaces. J Food Prot. 2012;75(4):701-705. doi:10.4315/0362-028X. JFP-11-276

15. Adeyemi OS, Molefe NI, Awakan OJ, et al. Metal nanoparticles restrict the growth of protozoan parasites. Artif Cells Nanomed Biotechnol. 2018;46(sup3):S86-S94. doi:10.1080/21691401.20 18.1489267

16. Colotti G, Ilari A, Fiorillo A, et al. Metal-based compounds as prospective antileishmanial agents: inhibition of trypanothione reductase by selected gold complexes. ChemMedChem. 2013;8 (10):1634-1637.

17. Ilari A, Baiocco P, Messori L, et al. A gold-containing drug against parasitic polyamine metabolism: the X-ray structure of trypanothione reductase from Leishmania infantum in complex with auranofin reveals a dual mechanism of enzyme inhibition. Amino Acids. 2012;42(2-3):803-811. doi:10.1007/s00726-011-0997-9

18. Baiocco P, Ilari A, Ceci P, et al. Inhibitory effect of silver nanoparticles on trypanothione reductase activity and Leishmania infantum proliferation. ACS Med Chem Lett. 2010;2(3):230-233. doi:10.1021/ ml1002629

19. Farajzadeh S, Parizi MH, Haghdoost AA, et al. Comparison between intralesional injection of zinc sulfate $2 \%$ solution and intralesional meglumine antimoniate in the treatment of acute old world dry type cutaneous leishmaniasis: a randomized double-blind clinical trial. $J$ Parasit Dis. 2016;40(3):935-939. doi:10.1007/s12639-014-0609-1

20. Ghasemi E, Ghaffarifar F, Dalimi A, Sadraei J. In-vitro and in-vivo antileishmanial activity of a compound derived of platinum, oxaliplatin, against Leishmania major. Iran J Pharm Res. 2019;18 (4):2028.

21. Afridi MS, Hashmi SS, Ali GS, Zia M, Abbasi BH. Comparative antileishmanial efficacy of the biosynthesised ZnO NPs from genus Verbena. IET Nanobiotechnol. 2018;12(8):1067-1073. doi:10.1049/ iet-nbt.2018.5076

22. Allahverdiyev AM, Abamor ES, Bagirova M, et al. Investigation of antileishmanial activities of Tio2@Ag nanoparticles on biological properties of $L$. tropica and $L$. infantum parasites, in vitro. Exp Parasitol. 2013;135(1):55-63. doi:10.1016/j.exppara.2013.06.001

23. Fanti JR, Tomiotto-Pellissier F, Miranda-Sapla MM, et al. Biogenic silver nanoparticles inducing Leishmania amazonensis promastigote and amastigote death in vitro. Acta Trop. 2018;178:46-54. doi:10.1016/j.actatropica.2017.10.027

24. Gold K, Slay B, Knackstedt M, Gaharwar AK. Antimicrobial activity of metal and metal-oxide based nanoparticles. Adv Ther. 2018;1 (3):1700033. doi:10.1002/adtp.201700033
25. Kong FY, Zhang JW, Li RF, Wang ZX, Wang WJ, Wang W. Unique roles of gold nanoparticles in drug delivery, targeting and imaging applications. Molecules. 2017;22(9):1445. doi:10.3390/molecules2 2091445

26. Torabi N, Mohebali M, Shahverdi AR, et al. Nanogold for the treatment of zoonotic cutaneous leishmaniasis caused by Leishmania major (MRHO/IR/75/ER): an animal trial with methanol extract of Eucalyptus camaldulensis. JPHS. 2011;1:15-18.

27. Sazgarnia A, Taheri AR, Soudmand S, Parizi AJ, Rajabi O, Darbandi MS. Antiparasitic effects of gold nanoparticles with microwave radiation on promastigotes and amastigotes of Leishmania major. Int $J$ Hyperth. 2013;29(1):79-86. doi:10.3109/ 02656736.2012 .758875

28. Jebali A, Anvari-Tafti MH. Hybridization of different antisense oligonucleotides on the surface of gold nanoparticles to silence zinc metalloproteinase gene after uptake by Leishmania major. Colloids Surf B Biointerfaces. 2015;129:107-113. doi:10.1016/j.colsurfb.20 15.03.029

29. Abamor ES, Allahverdiyev AM. A nanotechnology based new approach for chemotherapy of cutaneous leishmaniasis: TIO2@AG nanoparticles-Nigella sativa oil combinations. Exp Parasitol. 2016;166:150-163. doi:10.1016/j.exppara.2016.04.008

30. Corpas-López V, Díaz-Gavilán M, Franco-Montalbán F, et al. A nanodelivered Vorinostat derivative is a promising oral compound for the treatment of visceral leishmaniasis. Pharmacol Res. 2019;139:375-383. doi:10.1016/j.phrs.2018.11.039

31. Das S, Roy P, Mondal S, Bera T, Mukherjee A. One pot synthesis of gold nanoparticles and application in chemotherapy of wild and resistant type visceral leishmaniasis. Colloids Surf B Biointerfaces. 2013;107:27-34. doi:10.1016/j.colsurfb.2013.01.061

32. Das S, Halder A, Roy P, Mukherjee A. Biogenic gold nanoparticles against wild and resistant type visceral leishmaniasis. Mater Today: Proc. 2018;5(1):2912-2920.

33. Halder A, Das S, Bera T, Mukherjee A. Rapid synthesis for monodispersed gold nanoparticles in kaempferol and anti-leishmanial efficacy against wild and drug resistant strains. RSC Adv. 2017;7 (23):14159-14167. doi:10.1039/C6RA28632A

34. Berry SL, Hameed H, Thomason A, et al. Development of NanoLuc-PEST expressing Leishmania mexicana as a new drug discovery tool for axenic-and intramacrophage-based assays. PLoS Negl Trop Dis. 2018;12(7):e0006639. doi:10.1371/journal.pntd.0006639

35. Mahmoud AB, Danton O, Kaiser M, Khalid S, Hamburger M, Mäser P. HPLC-based activity profiling for antiprotozoal compounds in Croton gratissimus and Cuscuta hyalina. Front Pharmacol. 2020;11:1246. doi:10.3389/fphar.2020.01246

36. Ghosh S, Jagtap S, More P, et al. Dioscorea bulbifera mediated synthesis of novel Au core Ag shell nanoparticles with potent antibiofilm and antileishmanial activity. J Nanomater. 2015;16(1):161.

37. Botteon C, Silva L, Ccana-Ccapatinta G, et al. Biosynthesis and characterization of gold nanoparticles using Brazilian red propolis and evaluation of its antimicrobial and anticancer activities. Sci Rep. 2021;11(1):1-16. doi:10.1038/s41598-021-81281-w

38. Gatea F, Teodor ED, Seciu AM, et al. Antitumour, antimicrobial and catalytic activity of gold nanoparticles synthesized by different $\mathrm{pH}$ propolis extracts. J Nanopart Res. 2015;17(7):1-13. doi:10.1007/ s11051-015-3127-X

39. Want MY, Islammudin M, Chouhan G, et al. Nanoliposomal artemisinin for the treatment of murine visceral leishmaniasis. Int J Nanomed. 2017;12:2189. doi:10.2147/IJN.S106548

40. Tournebize J, Sapin-Minet A, Schneider R, Boudier A, Maincent P, Leroy P. Simple spectrophotocolorimetric method for quantitative determination of gold in nanoparticles. Talanta. 2011;83 (5):1780-1783. doi:10.1016/j.talanta.2010.12.005

41. Ramezani F, Jebali A, Kazemi B. A green approach for synthesis of gold and silver nanoparticles by Leishmania sp. Appl Biochem Biotechnol. 2012;168(6):1549-1555. doi:10.1007/s12010-012-9877-3 
42. Want MY, Islamuddin M, Chouhan G, Dasgupta AK, Chattopadhyay AP, Afrin F. A new approach for the delivery of artemisinin: formulation, characterization, and ex-vivo antileishmanial studies. J Colloid Interface Sci. 2014;432:258-269. doi:10.1016/ j.jcis.2014.06.035

43. Afrin F, Chouhan G, Islamuddin M, Want MY, Ozbak HA, Hemeg HA. Cinnamomum cassia exhibits antileishmanial activity against Leishmania donovani infection in vitro and in vivo. PLoS Negl Trop Dis. 2019;13(5):e0007227. doi:10.1371/journal. pntd.0007227

44. Chouhan G, Islamuddin M, Want MY, et al. Apoptosis mediated leishmanicidal activity of Azadirachta indicabioactive fractions is accompanied by Th1 immunostimulatory potential and therapeutic cure in vivo. Parasit Vectors. 2015;8(1):183. doi:10.1186/s13071015-0788-3

45. Mondal S, Roy P, Das S, Halder A, Mukherjee A, Bera T. In vitro susceptibilities of wild and drug resistant Leishmania donovani amastigote stages to andrographolide nanoparticle: role of vitamin E derivative TPGS for nanoparticle efficacy. PLoS One. 2013;8(12): e81492. doi:10.1371/journal.pone.0081492

46. Varshosaz J, Arbabi B, Pestehchian N, Saberi S, Delavari M. Chitosan-titanium dioxide-glucantime nanoassemblies effects on promastigote and amastigote of Leishmania major. Int J Biol Macromol. 2018;107:212-221. doi:10.1016/j.ijbiomac.2017.08.177

47. Vermeersch M, da Luz RI, Toté K, Timmermans J-P, Cos P, Maes L. In vitro susceptibilities of Leishmania donovanipromastigote and amastigote stages to antileishmanial reference drugs: practical relevance of stage-specific differences. Antimicrob Agents Chemother. 2009;53(9):3855-3859. doi:10.1128/AAC.00548-09

48. Dias CN, Nunes TAL, de Sousa JMS, et al. Methyl gallate: selective antileishmanial activity correlates with host-cell directed effects. Chem Biol Interact. 2020;320:109026.

49. De Muylder G, Ang KK, Chen S, Arkin MR, Engel JC, McKerrow JH. A screen against Leishmania intracellular amastigotes: comparison to a promastigote screen and identification of a host cell-specific hit. PLoS Negl Trop Dis. 2011;5(7):e1253. doi:10.1371/journal.pntd.0001253

50. De Rycker M, Hallyburton I, Thomas J, et al. Comparison of a high-throughput high-content intracellular Leishmania donovani assay with an axenic amastigote assay. Antimicrob Agents Chemother. 2013;57(7):2913-2922. doi:10.1128/AAC.02398-12

51. Yang Y, Matsubara S, Nogami M, Shi J, Huang W. One-dimensional self-assembly of gold nanoparticles for tunable surface plasmon resonance properties. Nanotechnology. 2006;17(11):2821. doi:10.1088/0957-4484/17/11/015

52. Zhang Q, Hitchins VM, Schrand AM, Hussain SM, Goering PL. Uptake of gold nanoparticles in murine macrophage cells without cytotoxicity or production of pro-inflammatory mediators. Nanotoxicology. 2011;5(3):284-295. doi:10.3109/17435390.20 10.512401

53. Xiang SD, Scholzen A, Minigo G, et al. Pathogen recognition and development of particulate vaccines: does size matter? Methods. 2006;40(1):1-9. doi:10.1016/j.ymeth.2006.05.016
54. Naha PC, Chhour P, Cormode DP. Systematic in vitro toxicological screening of gold nanoparticles designed for nanomedicine applications. Toxicol In Vitro. 2015;29(7):1445-1453. doi:10.1016/j. tiv.2015.05.022

55. França A, Aggarwal P, Barsov EV, Kozlov SV, Dobrovolskaia MA, González-Fernández Á. Macrophage scavenger receptor A mediates the uptake of gold colloids by macrophages in vitro. Nanomedicine. 2011;6(7):1175-1188. doi:10.2217/nnm.11.41

56. Godoy-Gallardo M, Eckhard U, Delgado LM, et al. Antibacterial approaches in tissue engineering using metal ions and nanoparticles: from mechanisms to applications. Bioact Mater. 2021;6 (12):4470-4490. doi:10.1016/j.bioactmat.2021.04.033

57. Cui Y, Zhao Y, Tian Y, Zhang W, Lü X, Jiang X. The molecular mechanism of action of bactericidal gold nanoparticles on Escherichia coli. Biomaterials. 2012;33(7):2327-2333. doi:10.1016/ j.biomaterials.2011.11.057

58. Alti D, Veeramohan Rao M, Rao DN, Maurya R, Kalangi SK. Goldsilver bimetallic nanoparticles reduced with herbal leaf extracts induce ROS-mediated death in both promastigote and amastigote stages of Leishmania donovani. ACS Omega. 2020;5 (26):16238-16245. doi:10.1021/acsomega.0c02032

59. Zhao P, Li T, Li Z, et al. Preparation of gold nanoparticles and its effect on autophagy and oxidative stress in chronic kidney disease cell model. J Nanosci Nanotechnol. 2021;21(2):1266-1271. doi:10.1166/jnn.2021.18655

60. Zhang W, Li Y, Niu J, Chen Y. Photogeneration of reactive oxygen species on uncoated silver, gold, nickel, and silicon nanoparticles and their antibacterial effects. Langmuir. 2013;29(15):4647-4651. doi:10.1021/la400500t

61. Chaloupka K, Malam Y, Seifalian AM. Nanosilver as a new generation of nanoproduct in biomedical applications. Trends Biotechnol. 2010;28(11):580-588. doi:10.1016/j.tibtech.2010.07.006

62. Shukla R, Bansal V, Chaudhary M, Basu A, Bhonde RR, Sastry M. Biocompatibility of gold nanoparticles and their endocytotic fate inside the cellular compartment: a microscopic overview. Langmuir. 2005;21(23):10644-10654. doi:10.1021/la0513712

63. Pan Y, Neuss S, Leifert A, et al. Size-dependent cytotoxicity of gold nanoparticles. Small. 2007;3(11):1941-1949. doi:10.1002/ smll.200700378

64. Isaac-Márquez A, Talamás-Rohana $\mathrm{P}$, Galindo-Sevilla $\mathrm{N}$, et al. Decanethiol functionalized silver nanoparticles are new powerful leishmanicidals in vitro. World J Microbiol Biotechnol. 2018;34 (3):38. doi:10.1007/s11274-018-2420-0

65. Kumar P, Shivam P, Mandal S, et al. Synthesis, characterization, and mechanistic studies of a gold nanoparticle-amphotericin B covalent conjugate with enhanced antileishmanial efficacy and reduced cytotoxicity. Int $J$ Nanomedicine. 2019;14:6073. doi:10.2147/IJN S196421
International Journal of Nanomedicine

\section{Publish your work in this journal}

The International Journal of Nanomedicine is an international, peerreviewed journal focusing on the application of nanotechnology in diagnostics, therapeutics, and drug delivery systems throughout the biomedical field. This journal is indexed on PubMed Central, MedLine, CAS, SciSearch ${ }^{\mathbb{R}}$, Current Contents ${ }^{\mathbb{B}} /$ Clinical Medicine,
Journal Citation Reports/Science Edition, EMBase, Scopus and the Elsevier Bibliographic databases. The manuscript management system is completely online and includes a very quick and fair peer-review system, which is all easy to use. Visit http://www.dovepress.com/ testimonials.php to read real quotes from published authors. 